\title{
CALIBRATION OF THE SR4500 TIME-OF-FLIGHT CAMERA FOR OUTDOOR MOBILE SURVEYING APPLICATIONS: A CASE STUDY
}

\author{
C. Heinkelé, M. Labbé, V. Muzet, P. Charbonnier
}

Cerema Est, Laboratoire de Strasbourg, 11 rue Jean Mentelin, B.P. 9, 67035 Strasbourg, France

\{Christophe.Heinkele,Valerie.Muzet,Pierre.charbonnier\}@cerema.fr, marionlabbe@ free.fr

Commission V, WG V/3

KEY WORDS: SR4500, ToF Cameras, Calibration, Outdoor dynamic acquisitions

\begin{abstract}
:
3D-cameras based on Time-of-Flight (ToF) technology have recently raised up to a commercial level of development. In this contribution, we investigate the outdoor calibration and measurement capabilities of the SR4500 ToF camera. The proposed calibration method combines up-to-date techniques with robust estimation. First, intrinsic camera parameters are estimated, which allows converting radial distances into orthogonal ones. The latter are then calibrated using successive acquisitions of a plane at different camera positions, measured by tacheometric techniques. This distance calibration step estimates two coefficient matrices for each pixel, using linear regression. Experimental assessments carried out with a 3D laser-cloud after converting all the data in a common basis show that the obtained precision is twice better than with the constructor default calibration, with a full-frame accuracy of about $4 \mathrm{~cm}$. Moreover, estimating the internal calibration in sunny and warm outdoor conditions yields almost the same coefficients as indoors. Finally, a test shows the feasibility of dynamic outdoor acquisitions and measurements.
\end{abstract}

\section{INTRODUCTION}

In this contribution, we study the metrological performance (setup and calibration) and the measurement capacities of a recent Timeof-Flight (ToF) camera, namely the SR4500, in the context of outdoor mobile surveying applications.

Range cameras have become considerably popular in the last decades, thanks to their ability to simultaneously acquire a range map and an intensity matrix, at a low cost and video frame rate. Thanks to the progress of technologies, they have raised up to a commercial level of development and are now routinely used in many applications, such as robotics, medicine, automobile or video gaming. Nowadays, industrial 3D cameras offer a cheap alternative, albeit less accurate, to laser scanners for short-range 3D surveying in controlled environments. However, the recent application of range cameras to outdoor 3D surveying at longer ranges (Chiabrando and Rinaudo, 2013, Lachat et al., 2015) raises the question of their metrological capabilities.

Range cameras based on ToF technologies have recently encountered an increasing attention, see e.g. (Piatti et al., 2013). Several industrial sensors were developed since the seminal work of (Lange, 2000), see e.g. (Piatti and Rinaudo, 2012) for a survey. In this contribution, we consider a phase modulated (or indirect ToF) camera, namely the SR4500 (Fig. 1), launched in 2014 and manufactured by Mesa Imaging (recently acquired by the Heptagon company). The sensor operates in the close infrared. It is an in-pixel photomixing device (Pancheri and Stoppa, 2013), i.e. it operates a demodulation of the received optical signal for each pixel.

While the former versions of the camera were limited to 5 meters, the SR4500 has the advantage of measuring up to $10 \mathrm{~m}$, depending on the acquisition frequency (frequencies may be set between 15 and $30 \mathrm{MHz}$ ). The integration time may be set in the range [0.3-25.8] ms. Its resolution is $176 \times 144$ pixels, for a field of

\footnotetext{
${ }^{*}$ Corresponding author
}

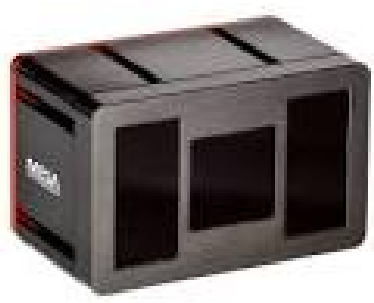

Figure 1. Picture of the SR4500 ToF camera

view of $69^{\circ} \times 55^{\circ}$. A sample acquisition is shown on Fig. 2. The scene is taken in a technical hall, with an optical bench and a test vehicle. Its depth varies from 0 to $10 \mathrm{~m}$. The left image shows the depth map (in false colors), where the depth gradient along the bench is clearly visible, along with some noisy pixels. The center image is the intensity image in the infrared which is, of course, slightly different from the rightmost photograph.

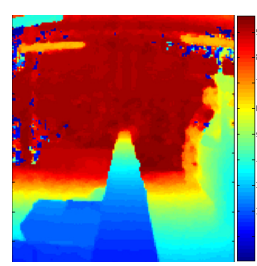

(a)

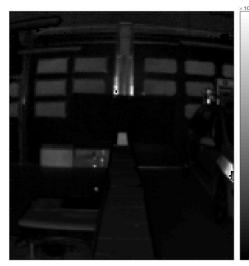

(b)

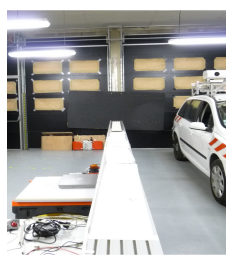

(c)
Figure 2. Sample acquisition of an indoor scene by the SR4500 ToF camera. (a) Distance matrix (b) amplitude matrix, in the close infrared (c) Photograph of the scene taken by an external camera

We have chosen the SR4500 camera because it is well suited to our research activity, that aims at developing tools for outdoor 
mobile surveying applications. More specifically, we want to survey objects (e.g. traffic signs) in urban road scenes, at a [3-8] m distance range, using dynamic acquisitions. In such a context, short integration times (typically less than $10 \mathrm{~ms}$ ) are required, at the price of more noisy acquisitions, which implies applying robust data processing methods.

In this paper, we first propose an introduction to the SR4500 camera and its behaviour (Sec. 2). It is clear that raw measurements are not accurate enough so a calibration is needed. In the literature, several publications report works on the calibration and metrological assessment of ToF cameras (in particular, of previous versions of SR cameras), see e.g. (Kahlmann et al., 2006, Jaakkola et al., 2008, Robbins et al., 2008, Chiabrando et al., 2009, Lefloch et al., 2013). Inspired by these papers, we introduce in Sec. 3 a two-step calibration methodology. Sec. 4 is dedicated to an experimental assessment of this method in laboratory and outdoor conditions. Finally, Sec. 5 is a feasibility study of dynamic outdoor acquisitions and measurements.

\section{BEHAVIOUR OF THE SR4500 CAMERA}

\subsection{Measurement principle}

The SR4500 processes each pixel using the continuous wave intensity modulation method, which is based on the correlation between the emitted signal and the received one (Lange, 2000). The emitted light being sinusoidal, with a fixed frequency $f$, the correlation function can be written, at $\tau=2 \pi f t$, as (Lefloch et al., 2013):

$$
c_{\tau}=A_{0}+\frac{A}{2} \cos (\tau+\varphi)
$$

where $A$ is the amplitude of the signal, $A_{0}$ is the offset and $\varphi$ is the phase shift between the emitted and received waves. Since there are 3 unknowns, at least 3 values are needed to calculate these parameters. Typically, 4 samples are used, at specific phase values, $\tau=0, \pi / 2, \pi, 3 \pi / 2$, as illustrated in Fig. 4 .

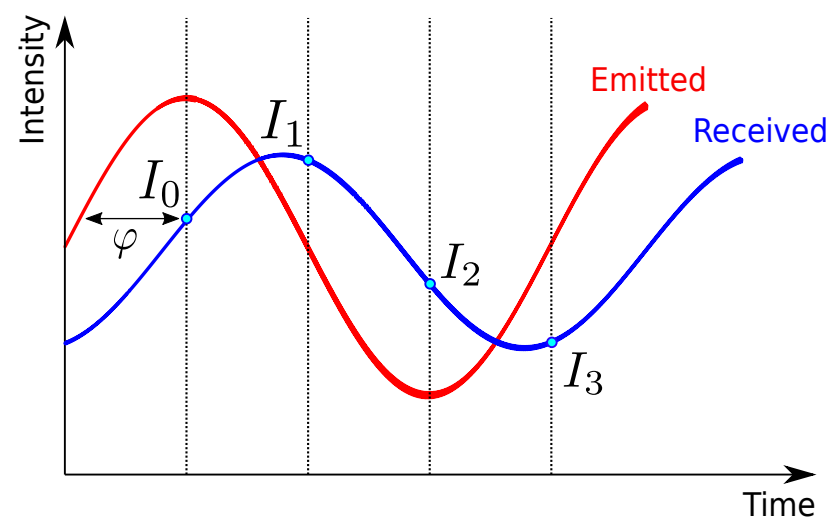

Figure 3. Overview of phase determination technique

The phase, amplitude and offset parameters are respectively given by:

$$
\begin{gathered}
\varphi=\arctan \frac{I_{3}-I_{1}}{I_{0}-I_{2}} \\
A=\frac{1}{2} \sqrt{\left(I_{3}-I_{1}\right)^{2}+\left(I_{0}-I_{2}\right)^{2}} \\
A_{0}=\frac{\left(I_{0}+I_{1}+I_{2}+I_{3}\right)}{4}
\end{gathered}
$$

The distance to the observed object (i.e. the depth) is related to the observed signal phase by the following formula

$$
d=\frac{c \varphi}{4 \pi f}
$$

where $c$ is the speed of light. As for all modulated methods, this formula is valid up to a maximum phase shift of $\varphi=2 \pi$. We will use an acquisition frequency of $15 \mathrm{MHz}$, for which the maximal reachable distance is $10 \mathrm{~m}$, which is well suited to our application.

In the SR4500, 4 separate integration periods are needed to take the 4 samples that are necessary for phase measurement (Mesa Imaging AG, n.d.). The total time required to capture a depth image is hence 4 times the integration time (i.e. the time interval during which intensities are collected by the sensor) plus the readout time (i.e. the amount of time required to read the values). While the readout time is incompressible (about $4.6 \mathrm{~ms}$ for the SR4500), the integration time is a parameter, which can strongly influence distance measurements. Too high values may lead to saturation effects, while too low ones may cause noise. Moreover, it was shown in (Kahlmann et al., 2006) and (Pfeifer et al., 2013) that the integration time may result in distance errors in the order of $10 \mathrm{~cm}$. However, application-based constraints can influence the way this value is set. In our case, dynamic acquisitions are envisioned, so short integrations times are needed to avoid motion blur and typical values of 3.3 to $10 \mathrm{~ms}$ will be taken.

\subsection{Quality of ToF data}

The raw measurements provided by ToF cameras are impaired by errors of different natures. A comprehensive panorama of rangecamera errors is out of the scope of this paper and we refer the reader to e.g. (Lefloch et al., 2013, Rapp, 2007) for more insight about these phenomena. In this section, we illustrate systematic and random errors on a simple example.

The repeatability of the raw data of the SR 4500 was studied with 50 acquisitions of a white wall placed at $2 \mathrm{~m}$ of the camera. The integration time was set at $3.3 \mathrm{~ms}$. The standard deviation of these 50 measures was computed for each pixel and is shown in Fig. 4. In the central part of the image, the standard deviation is between 2 and $3 \mathrm{~cm}$ and it reaches $9 \mathrm{~cm}$ in the corners. We note that these results are qualitatively similar to those obtained by Kahlman (Kahlmann et al., 2006) for a SR-2 camera.

The same experiment was done for a $10 \mathrm{~ms}$ integration time. With this setting, the standard deviation of the center is around $0.72 \mathrm{~cm}$ while it is $4 \mathrm{~cm}$ on the borders. We observe that the standard deviation decreases when the integration time is bigger. This is in accordance with the variation law of the variance with respect to the inverse of the squared amplitude exhibited by Rapp in (Rapp, 2007).

This example illustrate the need for correcting measurement errors. To reduce the effect of random errors, averages are often used. However, it is most of the time preferable to use more robust estimators, such as the median, for example. Systematic measurement errors may be addressed by calibration.

\subsection{Internal heating time and integration time}

As all photonic devices, range camera sensor needs time to reach stability (Kahlmann et al., 2006, Rapp, 2007). For example, with integration times between 20 and $110 \mathrm{~ms}$, the SR4000 needs about 40 minutes to reach stability (Chiabrando et al., 2009). In this experiment, we studied the internal heating time of the SR4500, at 


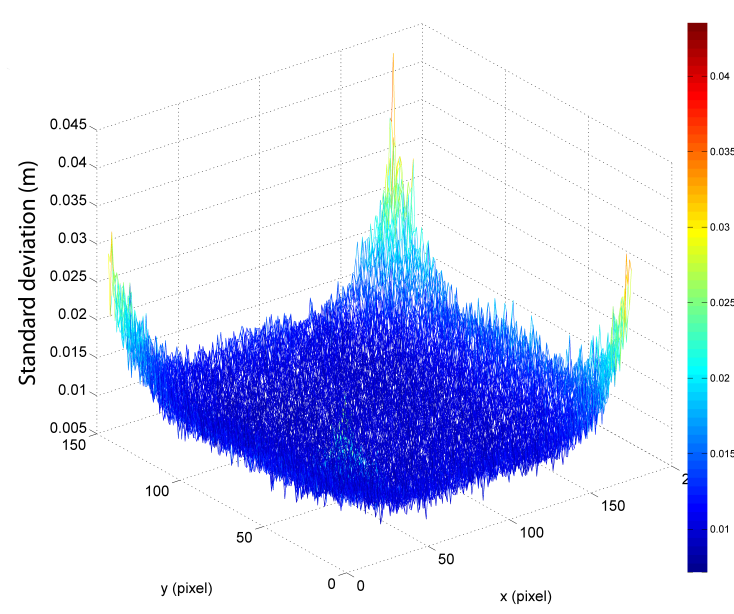

Figure 4. Standard deviation $(\mathrm{m})$ of 50 acquisitions (sight distance of $2 \mathrm{~m}$, integration time of $3.3 \mathrm{~ms}$ )

$$
\begin{array}{|cc|}
\hline \square & 3.3 \mathrm{~ms} \\
\square & 5.3 \mathrm{~ms} \\
\square & 10 \mathrm{~ms} \\
\square & 20 \mathrm{~ms} \\
& \text { Real distance }
\end{array}
$$

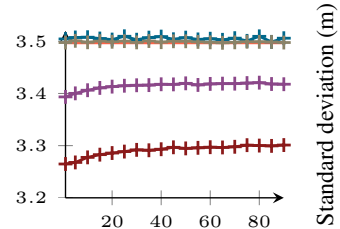

Time (min)

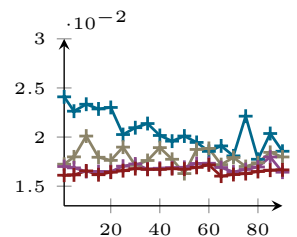

Time (min)
Figure 5. Evolution of the measured distance (left) and standard deviation (right) with time for different acquisition times

a sight distance of $3.5 \mathrm{~m}$ for 4 different integration time: $3.3,5.3$, 10 and $20 \mathrm{~ms}$. For each integration time, 50 acquisitions were taken every $5 \mathrm{~min}$ and we studied the raw data given by the Mesa software. The median and the standard deviation are presented for the central part of the image $(30 \times 24$ pixels $)$ in Fig. 5 . The measured distance almost corresponds to the real distance at 3.3 and $5.5 \mathrm{~ms}$ while there is an important distance offset at 10 and $20 \mathrm{~ms}$. Since the default value of the Mesa SR4500 integration time is $3.3 \mathrm{~ms}$ (Mesa Imaging AG, n.d.), it seems that the default calibration corresponds to this setting. For the longest integration times of our study ( 10 and $20 \mathrm{~ms}$ ), during the first 20 minutes of heating, the variation of the measured distance reaches $4 \mathrm{~cm}$. At an acquisition time of $3.3 \mathrm{~ms}$, there is less variation of the median distance but the standard deviation needs more time to reach stability (40 to 60 minutes). Hence, a heating time of $40 \mathrm{~min}$ at least was systematically respected in all our experiments.

This experiment confirms the need for a correction of distances. Clearly, this calibration must be adapted to each integration time setting.

\section{CALIBRATION METHODOLOGY}

Sources of systematic error are many in range imaging, see e.g. (Lefloch et al., 2013). In such a context, substantial gains in precision are accessible using calibration techniques. In this section,

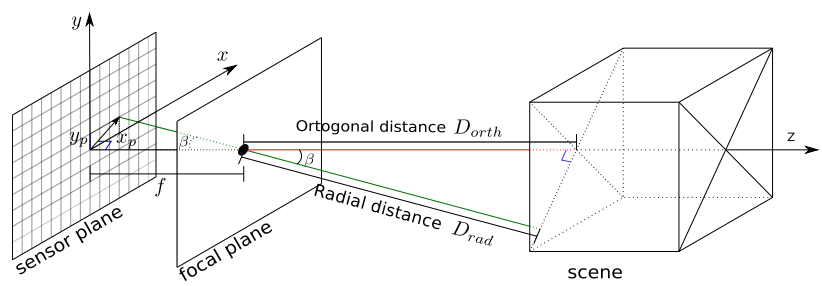

Figure 6. Transforming a radial distance into an orthogonal distance, following (Rapp et al., 2008)

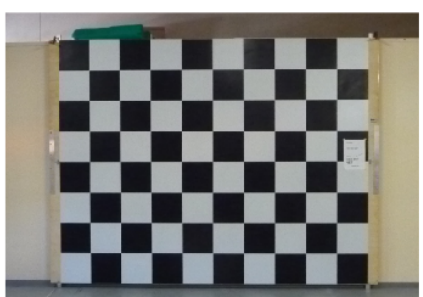

(a)

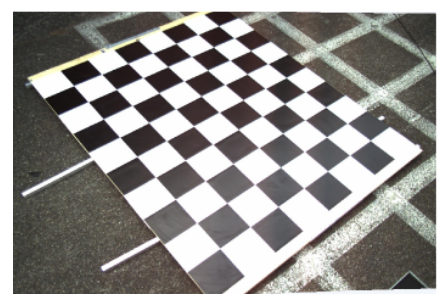

(c)

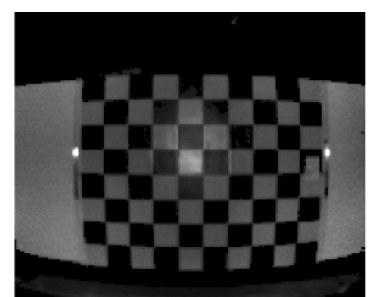

(b)

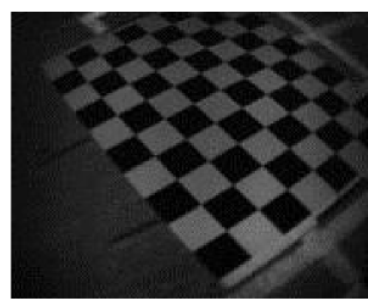

(d)
Figure 7. Photographs of the calibration checkerboard installed indoors (a) and outdoors (c); corresponding amplitude images output by the SR4500 indoors (b) and outdoors(d)

we recall the basis ideas of the two calibration procedures we use. The first one, internal, or lateral (Lindner and Kolb, 2006), calibration aims at transforming the radial distance provided by the range camera into an orthogonal distance, or depth. The second one, distance or depth calibration, is used to adjust the outputs to reference measurements.

\subsection{Internal calibration}

As for ordinary cameras, the internal calibration of range cameras relies on the determination of the intrinsic parameters of the device. These camera-specific parameters are, in particular, the focal length $f$, the optical image center $\left(c_{x}, c_{y}\right)$, the pixel size and the distortion coefficients. Once these parameters are estimated, it is possible to compensate for distortions caused by the sensor lens, but also to convert any radial distance $D_{\text {rad }}$ into an orthogonal one $D_{\text {orth }}$ using the pin-hole model, as described e.g. in (Rapp et al., 2008), see Fig. 6. The transformation is governed by the following relationship:

$$
D_{\text {orth }}=D_{\text {rad }} \cdot \cos \left(\arctan \left(\frac{\sqrt{x_{p}^{2}+y_{p}^{2}}}{f}\right)\right)
$$

where $\left(x_{p}, y_{p}\right)$ are the coordinate of the point in the image plane.

Among the multiple available calibration packages, we have chosen Bouguet's Matlab ${ }^{\circledR}$ calibration toolbox (Bouguet, 1999). Several views of a specially designed calibration checkerboard (see Fig. 7), whose dimensions $(3 \times 2.4 \mathrm{~m})$ are well suited to our application, are taken. More specifically, the corresponding amplitude matrices provided by the SR 4500 are used as data inputs. In practice we do not use all the parameters output by Bouguet's 
calibration routine. For example, we found that the focal distance was almost the same along both axes, and that the skew angle and tangential distortion coefficients could be neglected. Moreover, we consider a fourth-order radial distortion model, due to the negligible influence of the sixth order.

\subsection{Distance calibration}

The second step consists of the calibration of the orthogonal distances obtained from eq. (6). Successive images of a plane surface are taken with the SR4500 at different distances, as illustrated on Fig. 8. These reference distances are measured with a very accurate system (such as laser scanners, tacheometers (Chiabrando et al., 2009) or linear positionner tables (Rapp, 2007)).

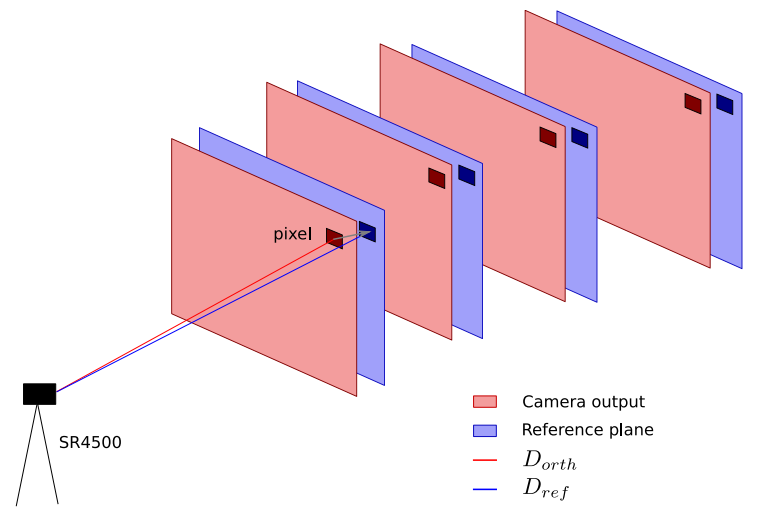

Figure 8. Scheme of the distance calibration method: reference distances are drawn in blue and SR4500 outputs are in red

In this work, we perform a per-pixel linear calibration. In other words, a linear regression is computed for each pixel, to adjust the data given by the camera to the reference distances. The output of the procedure is hence a pair of 25344-element matrices: one for the slopes and one for the offsets. Other techniques might be used, such as lookup tables (Kahlmann et al., 2006), but linear adjustment is more storage-efficient, as noticed in (Lindner and Kolb, 2006). While more sophisticated models, involving Bspline global correction (Lindner and Kolb, 2006) or polynomial regression (Schiller et al., 2008), might be envisioned, we found that the linear model was a good compromise between accuracy and complexity for our applications.

\section{EXPERIMENTAL CALIBRATIONS}

In this section, we propose an experimental assessment of the calibration methodology in both indoor and outdoor conditions.

\subsection{Internal calibration}

The calibration checkerboard has been used indoors and outdoors. In the indoor setting it is fixed on a wall and the camera is placed on a tripod; outdoors, it is laid on the floor and the SR4500 is attached to a forklift as depicted on Fig. 9. Figure 10 shows the position and orientation of the views taken in both situations.

Table 1 shows a comparison of the intrinsic parameters obtained in both configurations. Although the uncertainties provided by Bouguet's toolbox significatively increased outdoors, the differences of the computed parameters are quite small.

Despite the experimental conditions were not perfect (in particular, the ground is not really plane), this experiment shows the feasibility of an internal calibration outdoors, which might be of a great interest for future applications.

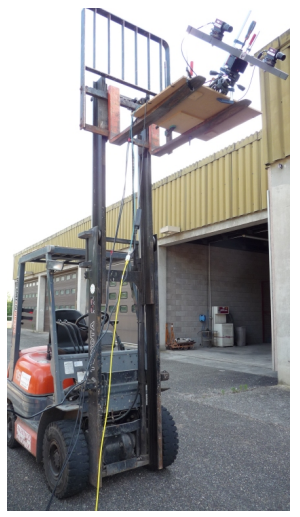

Figure 9. The SR4500 is fixed on a forklift for outdoor internal calibration

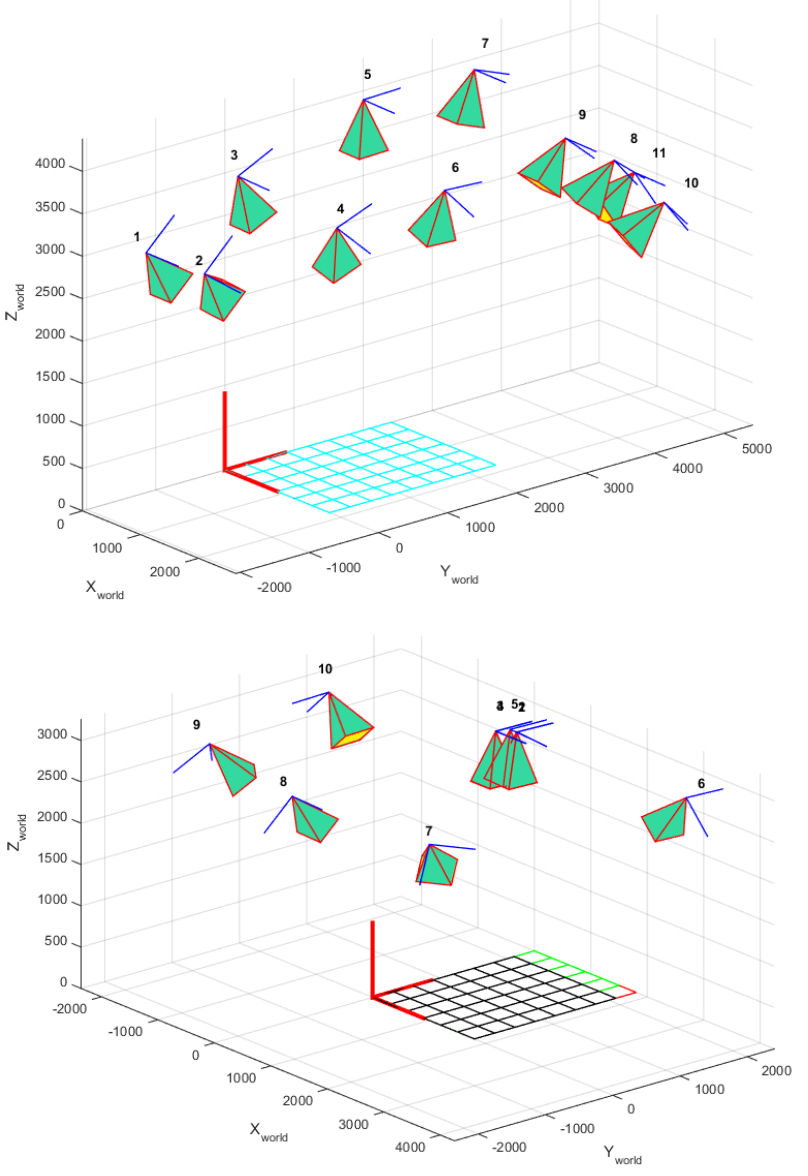

Figure 10. Orientation of the camera during indoor calibration (top) and outdoor calibration (bottom)

\begin{tabular}{|c|c|c|c|c|}
\hline & \multicolumn{2}{|c|}{ Indoor calibration } & \multicolumn{2}{c|}{ Outdoor calibration } \\
\hline & values & uncertainties & values & uncertainties \\
\hline$f_{x}$ & 10.09 & 0.09 & 10.08 & 0.18 \\
$f_{y}$ & 10.09 & 0.08 & 10.08 & 0.19 \\
\hline$c_{x}$ & 3.61 & 0.12 & 3.62 & 0.11 \\
$c_{y}$ & 2.65 & 0.11 & 2.72 & 0.17 \\
\hline
\end{tabular}

Table 1. Comparison of the SR4500 intrinsic parameters in $\mathrm{mm}$ between indoor and outdoor calibration 


\subsection{Distance calibration}

The calibration of the orthogonal distances is computed from successive acquisitions of a plane for different camera positions. These successive planes (Fig. 8) are obtained by moving the camera and making acquisition of a wall. At each scanning position, two Trimble M3 tachometers with a measurement precision of $3 \mathrm{~mm}$ are used for locating the camera and measuring its distance from the observed wall. To make the absolute localisation of the SR4500 ToF camera easier, several stickers are applied on it (see Fig. 11). To find the stickers coordinates and the checkerboards centres in the world scene, the tachometer data are processed with the Autocad COVADIS plug-in. Four checkerboards are also placed on the wall (Fig. 12) and their four centers are acquired by each tachometer.

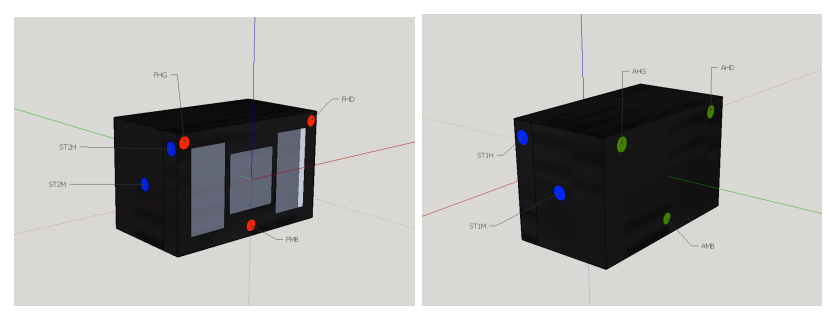

Figure 11. Stickers applied on the SR4500 camera to facilitate its localisation with the tachometer

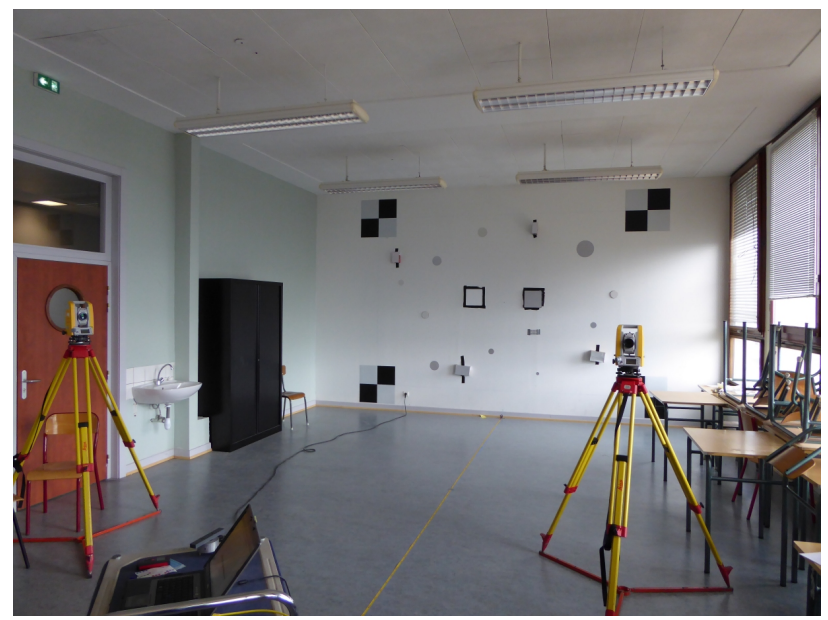

Figure 12. View of the calibration wall and the 2 tachometers

At each of 22 scanning position, 50 images are taken by the SR4500 with a $3.3 \mathrm{~ms}$ integration time and their median is computed. These data are then used for per-pixel linear regression with respect to the reference plane positions.

A crucial point for the method is to measure the reference planes accurately. Once 3 points on the camera and 3 points on the wall are known in world coordinates, it is possible to estimate the 3D Helmert transformation between the camera and the reference plane. However, the stickers applied on the ToF camera are too close to each others, and the obtained precision is not sufficient, especially on the rotational components of the transform. Fig. 13(a) shows that the reference and measured planes, that should be parallel, are indeed secant. To fix the reference plane orientation problem, we estimate the rotational components using the normal to each plane. Since the SR4500 data are very noisy, the normal to the camera point cloud is estimated using a robust Principal Component Analysis (PCA) algorithm, see (Moisan et al., 2015) for more details.

Once the orientation is corrected, the planes are parallel, as shown

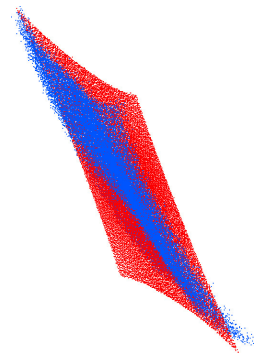

(a)

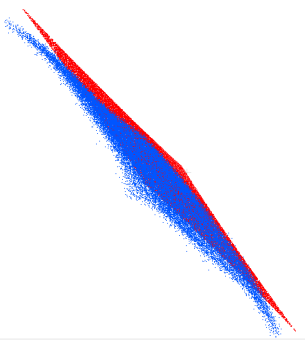

(b)
Figure 13. Point cloud of the calibration plane generated by the camera (in blue) and reference plane (in red). (a) obtained by Helmert 3D transformation (b) after alignment of the normal to the planes

in Fig. 13(b). Finally, a linear regression is performed on each pixel between the reference planes and the ToF camera cloud. As planned, the regression is linear and 2 matrices of $144 \times 176=$ 25344 coefficients are computed, one for the slope and one for the offset. After applying these coefficients to each acquisition, a depth map is computed for each acquisition distance.

\subsection{Accuracy and dispersion assessments}

In order to assess the quality of our calibration, a very accurate survey of the wall was performed using a Faro ${ }^{\circledR}$ Focus3D. The resolution of the resulting geo-referenced point cloud is $2 \mathrm{~mm}$. It is then down-sampled to the SR4500 resolution. The RootMean-Square (RMS) deviation is computed between the reference FARO plane and the depth maps. Fig. 14 shows the RMS deviation as a function of the sight distance, for both the depth maps provided by the Mesa-Imaging software, which uses the internal default calibration of the camera, and the depth maps obtained by our method. The accuracy is about $4 \mathrm{~cm}$ with our calibration and $6 \mathrm{~cm}$ for the Mesa output.

To estimate the dispersion of the data points, a plane is first adjusted to the depth maps by robust PCA and the standard deviation of the depths with respect to this plane is computed. Fig. 15 shows the obtained precision as a function of the sight distance, for the Mesa-imaging depth maps and the results of our method. At $4.96 \mathrm{~m}$, the mean standard deviation obtained with our calibration is $\pm 1.6 \mathrm{~cm}$ instead of $\pm 3.3 \mathrm{~cm}$ for the default calibration. The proposed method reduces systematic errors better than the Mesa-Imaging software.

\section{FIRST ATTEMPT AT OUTDOOR DYNAMIC ACQUISITIONS}

Since our laboratory results were satisfactory, a dynamic acquisition test was performed outdoors, at slow motion. The object we survey is a white grid (with spacing $1 \mathrm{~m} \pm 2 \mathrm{~cm}$ ) painted on a contrasting road surface, with an almost horizontal profile (see Fig. 16). For practical reasons, the acquisitions were performed with the constructor software SR3DView and, in this first approach, we used the default calibration given by Mesa.

\subsection{Experimental set-up}

Figure 17 summarizes the experimental setup. The ToF camera is fixed on a forklift at $2.5 \mathrm{~m}$ from the ground with a sight angle of $50^{\circ}\left( \pm 10^{\circ}\right)$. In order to reduce the number of outliers (flying pixels), the chosen integration time is $10 \mathrm{~ms}$, which yields 18 images per second. The experiment took place in hot temperature conditions $\left(35^{\circ} \mathrm{C}\right)$ and the sun illumination was about 60000 lux. 


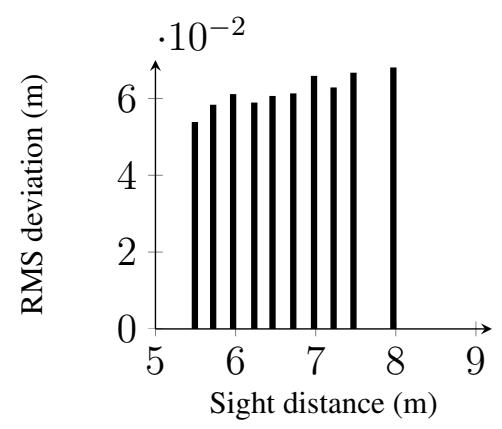

(a)

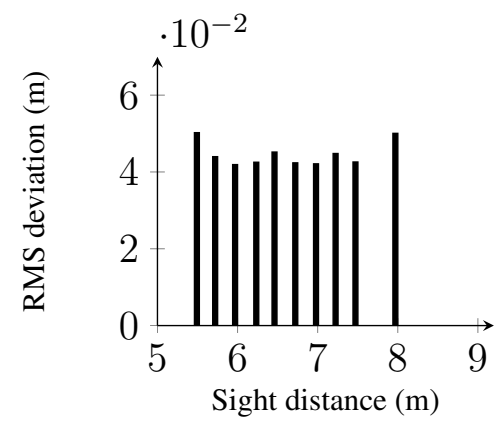

(b)

Figure 14. RMS deviation (accuracy) at different observation distances (a) between the depth maps obtained by Mesa-Imaging software and the Faro plane, (b) between the corrected depths obtained with our distance calibration and the FARO plane

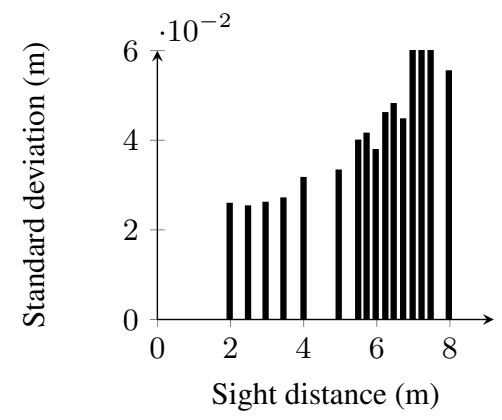

(a)

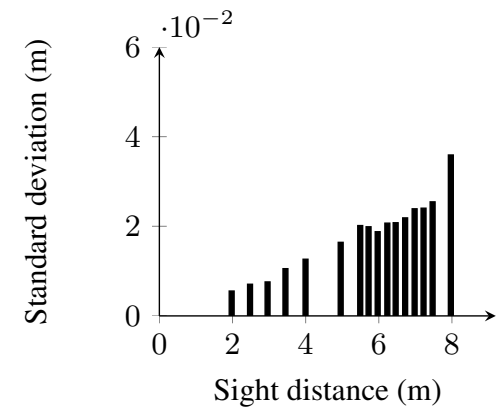

(b)

Figure 15. Standard deviation (precision) at different observation distances for (a) the Mesa-Imaging software (b) our calibration method

The image recording was done while the forklift moved at about $4 \mathrm{~km} \cdot \mathrm{h}^{-1}$

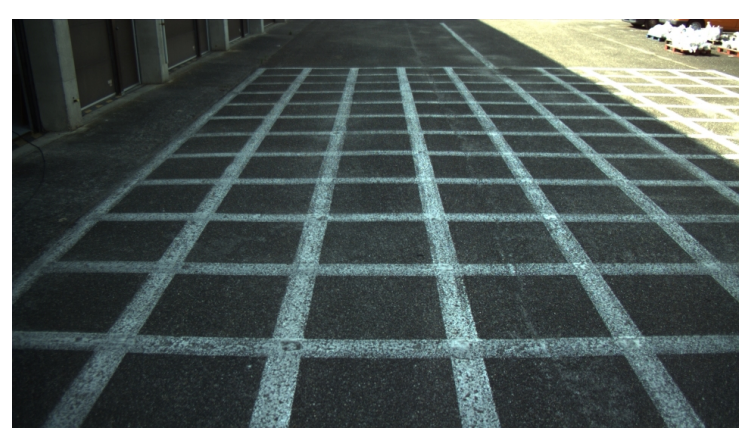

Figure 16. Picture of the surface of interest
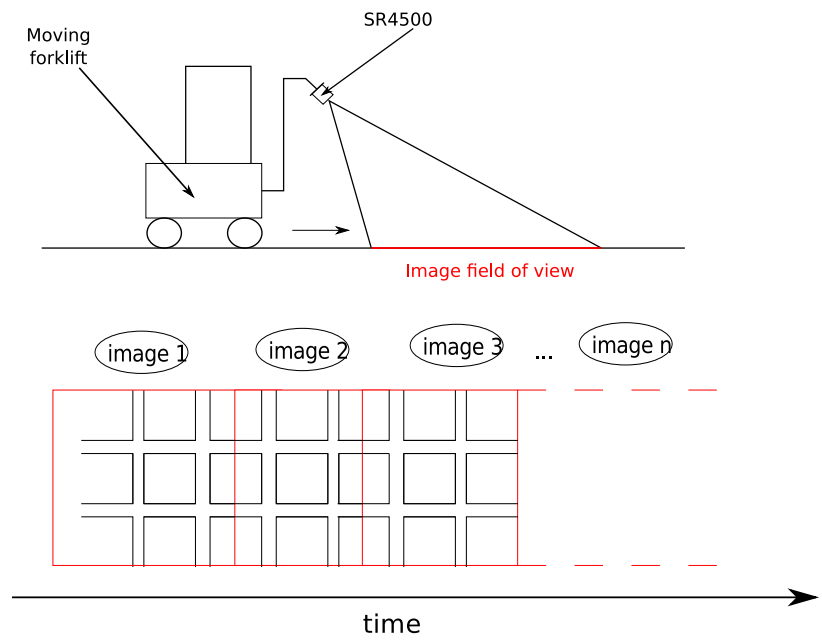

Figure 17. Sketch of the experimental outdoor set-up in dynamic

\subsection{Points cloud processing}

The point clouds provided by the camera are very noisy, as can be seen on Fig. 18. However, they can be easily enhanced by taking advantage of prior information about the scene and accounting for the amplitude image. Indeed, most flying pixels correspond to low amplitudes, so they can be eliminated by a simple threshold. The remaining outliers are filtered out after fitting the ground plane using robust PCA.

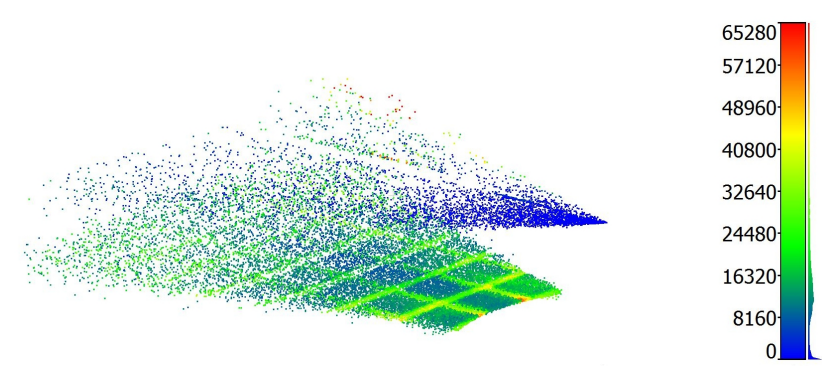

Figure 18. Point cloud of one acquisition of the grid. Point colors depict the amplitude signal. Outliers pixels are mostly located on a plane above the road

Then, a manual registration is performed using correspondances between grid corners in successive amplitude images. This yields a rough initial global model, which is refined by successive applications of the Iterative Closest Points (ICP) algorithm (Besl and McKay, 1992). A sample result is shown in Fig. 19. Note that most treatments are done manually for the moment because only a dozen of clouds have to be merged. With bigger scenes or more images, we should develop an automatic procedure. 


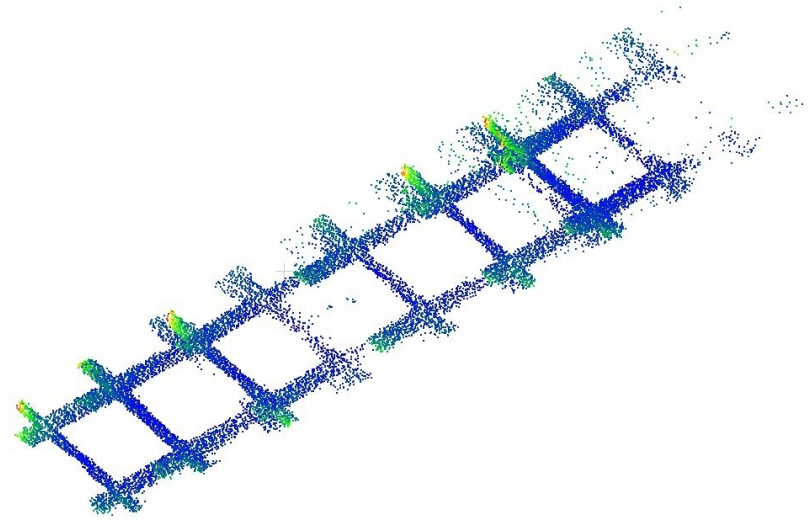

Figure 19. Full grid point cloud reconstructed by successive ICP

To assess the feasibility of dimensional measurements in these conditions we calculate the size of the grid marking. Since the contrast between the marking and the asphalt is important, the scalar information of the amplitude image output by the ToF camera can be used. The marking is segmented using an arbitrary threshold. Then, rectangles are adjusted in 3D using some selected points, see 20 . Their sizes can be compared with the physical size of the marking, as shown on Tab. 2 (the theoretical size is $19 \times 81 \mathrm{~cm})$.

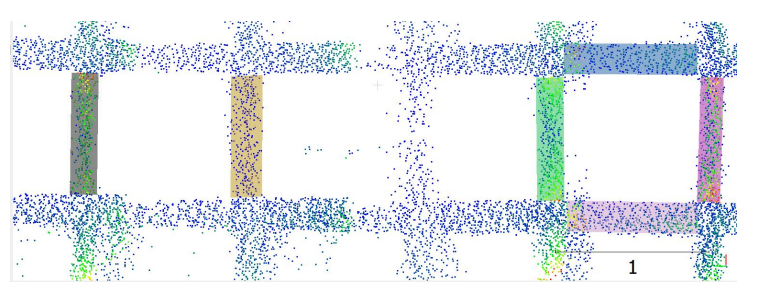

Figure 20. Extraction of several rectangles (successively colored in violet, green, blue, orange, pink and black) from the SR4500 point cloud that correspond to the marking

\begin{tabular}{|c|c|}
\hline Colour & Width $(\mathrm{cm}) \times$ length $(\mathrm{cm})$ \\
\hline violet & $16 \times 81$ \\
\hline green & $20 \times 81$ \\
\hline blue & $21 \times 87$ \\
\hline orange & $21 \times 79$ \\
\hline pink & $20 \times 85$ \\
\hline black & $18 \times 79$ \\
\hline
\end{tabular}

Table 2. Measured size of marking extracted from the local planes represented in colours on figure 20

With this methodology, the precision of marking sizes is around $2 \mathrm{~cm}$, which corresponds to the precisions of the grid. Since the data were taken at low speed, under a high sun illumination, and without specific calibration, these results are promising.

\section{CONCLUSION}

In this paper, we first presented the two calibration steps of the SR4500 ToF camera. The internal calibration was done indoors and outdoors, whereas the distance calibration was performed only indoors. Our experimental full-frame assessments show an accuracy of about $4 \mathrm{~cm}$ with a precision of $1.6 \mathrm{~cm}$ at $5 \mathrm{~m}$.

This study reveals a very good behaviour of the SR4500 outdoors, even in hot temperature conditions and high illumination level.
The internal camera parameters estimated in these sunny conditions are similar to those obtained indoors. A perspective of the present work could be to perform a distance calibration outdoors with a scanner laser acquisition of the whole scene at each camera position. With this setup, the reference could be more accurate and better accuracies may be expected.

Finally, the experiments show the feasibility of using the SR4500 ToF Camera outdoors even for dynamic applications, albeit the speed was limited in our experiments. Up to now, the ToF Camera cloud are processed manually. Further experiments in dynamic conditions will need more automated processes.

\section{REFERENCES}

Besl, P. and McKay, N. D., 1992. A method for registration of 3-D shapes. IEEE Transactions on Pattern Analysis and Machine Intelligence, 14(2), pp. 239-256.

Bouguet, J.-Y., 1999. Camera Calibration Toolbox for Matlab, http://www.vision.caltech.edu/bouguetj/calib_doc/index.html. Accessed April 2016.

Chiabrando, F. and Rinaudo, F., 2013. TOF Cameras for Architectural Surveys. In: F. Remondino and D. Stoppa (eds), TOF Range-Imaging Cameras, Springer Berlin Heidelberg, Berlin, Heidelberg, pp. 139-164.

Chiabrando, F., Chiabrando, R., Piatti, D. and Rinaudo, F., 2009. Sensors for 3D imaging: metric evaluation and calibration for a CCD/CMOS time-of-flight camera. Sensors, 9, pp. 1008010096.

Jaakkola, A., Kaasalainen, S., Hyyppä, J., Akujärvi, A. and Niittymäki, H., 2008. Radiometric calibration of intensity images of swissranger SR-3000 range camera. The photogrammetric journal of Finland, 21(1), pp. 16-25.

Kahlmann, T., Remondino, F. and Ingensand, H., 2006. Calibration for increased accuracy of the range imaging camera Swissranger. In: H.-G. Maas and D. Schneider (eds), Proceedings of the ISPRS Commission V Symposium 'Image Engineering and Vision Metrology', IAPRS Volume XXXVI, Part 5, Dresden, Germany, pp. 136-141.

Lachat, E., Macher, H., Mittet, M.-a., Landes, T. and Grussenmeyer, P., 2015. First Experiences With Kinect V2 Sensor for Close Range 3D Modelling. ISPRS - International Archives of the Photogrammetry, Remote Sensing and Spatial Information Sciences, XL-5/W4(February), pp. 93-100.

Lange, R., 2000. 3D time-of-flight distance measurement with custom solid-state image sensors in CMOS/CCD-technology. $\mathrm{PhD}$ thesis, Siegen.

Lefloch, D., Nair, R., Lenzen, F., Schaefer, H., Streeter, L., Cree, M. J., Koch, R. and Kolb, A., 2013. Time-of-Flight and Depth Imaging. Sensors, Algorithms, and Applications. Vol. 8200, Springer Berlin Heidelberg, chapter Technical Foundation and Calibration Methods for Time-of-Flight Cameras, pp. 3-24.

Lindner, M. and Kolb, A., 2006. Lateral and Depth Calibration of PMD-Distance Sensors. Advances in Visual Computing, 4292(4292/2006), pp. 524-533.

Mesa Imaging AG, n.d. SR4000/SR4500 User Manual. Technoparkstrasse 1, 8005 Zurich. Version 3.0. 
Moisan, E., Charbonnier, P., Foucher, P., Grussenmeyer, P., Guillemin, S. and Koehl, M., 2015. Adjustment of sonar and laser acquisition data for building the 3D reference model of a canal tunnel. Sensors, 15(12), pp. 31180-31204. Special Issue Sensors and Techniques for 3D Object Modeling in Underwater Environments.

Pancheri, L. and Stoppa, D., 2013. TOF Cameras for Architectural Surveys. In: F. Remondino and D. Stoppa (eds), Sensors based on in-pixel photomixing devices, Springer Berlin Heidelberg, Berlin, Heidelberg, pp. 69-90.

Pfeifer, N., Lichti, D., Böhm, J. and Karel, W., 2013. 3D Cameras: Errors, Calibration and Orientation. In: F. Remondino and D. Stoppa (eds), TOF Range-Imaging Cameras, Springer Berlin Heidelberg, pp. 117-138.

Piatti, D. and Rinaudo, F., 2012. SR-4000 and camcube3.0 time of flight (ToF) cameras: Tests and comparison. Remote Sensing, 4, pp. 1069-1089.

Piatti, D., Remondino, F. and Stoppa, D., 2013. TOF Cameras for Architectural Surveys. In: F. Remondino and D. Stoppa (eds), State-of-the art of TOF Range-Imaging sensors, Springer Berlin Heidelberg, Berlin, Heidelberg, pp. 1-10.
Rapp, H., 2007. Experimental and theoretical investigation of correlating TOF-camera systems. Thesis in physics, Insitut $\mathrm{f}$ ur Wissenschaftliches Rechnen (IWR), University of Heidelberg, Germany.

Rapp, H., Frank, M., Hamprecht, F. and Jahne, B., 2008. A theoretical and experimental investigation of the systematic errors and statistical uncertainties of Time-Of-Flight-cameras. International Journal of Intelligent Systems Technologies and Applications, 5, pp. 402-413.

Robbins, S., Schroeder, B., Murawski, B., Heckman, N. and Leung, J., 2008. Photogrammetric calibration of the swissranger 3D range imaging sensor. In: Proceedings of SPIE, Vol. 7003number 700320, SPIE Digital Library.

Schiller, I., Beder, C., and Koch, R., 2008. Calibration of a PMD camera using a planar calibration object together with a multi-camera setup. In: The International Archives of the Photogrammetry, Remote Sensing and Spatial Information Sciences, Vol. XXXVII. Part B3a, ISPRS, Beijing, China, pp. 297-302. 